\title{
Follow-up of Blood-Pressure Lowering and Glucose Control in Type 2 Diabetes
}

\author{
S. Zoungas, J. Chalmers, B. Neal, L. Billot, Q. Li, Y. Hirakawa, H. Arima, \\ H. Monaghan, R. Joshi, S. Colagiuri, M.E. Cooper, P. Glasziou, D. Grobbee, \\ P. Hamet, S. Harrap, S. Heller, L. Lisheng, G. Mancia, M. Marre, D.R. Matthews, \\ C.E. Mogensen, V. Perkovic, N. Poulter, A. Rodgers, B. Williams, S. MacMahon, \\ A. Patel, and M. Woodward, for the ADVANCE-ON Collaborative Group*
}

ABSTRACT

The authors' full names, academic degrees, and affiliations are listed in the Appendix. Address reprint requests to $\mathrm{Dr}$. Zoungas at the George Institute for Global Health, P.O. Box M201, Missenden Rd., Camperdown, NSW 2050, Australia.

Drs. Zoungas and Chalmers, and Drs. Patel and Woodward, contributed equally to this article.

*A complete list of members of the Action in Diabetes and Vascular Disease: Preterax and Diamicron Modified Release Controlled Evaluation Observational Study (ADVANCE-ON) Collaborative Group is provided in the Supplementary Appendix, available at NEJM.org.

This article was published on September 19, 2014, at NEJM.org.

N Engl J Med 2014;371:1392-406. DOI: 10.1056/NEJMoal407963

Copyright (๑ 2014 Massachusetts Medical Society.

\section{BACKGROUND}

In the Action in Diabetes and Vascular Disease: Preterax and Diamicron Modified Release Controlled Evaluation (ADVANCE) factorial trial, the combination of perindopril and indapamide reduced mortality among patients with type 2 diabetes, but intensive glucose control, targeting a glycated hemoglobin level of less than $6.5 \%$, did not. We now report results of the 6-year post-trial follow-up.

\section{METHODS}

We invited surviving participants, who had previously been assigned to perindopril-indapamide or placebo and to intensive or standard glucose control (with the glucose-control comparison extending for an additional 6 months), to participate in a post-trial follow-up evaluation. The primary end points were death from any cause and major macrovascular events.

RESULTS

The baseline characteristics were similar among the 11,140 patients who originally underwent randomization and the 8494 patients who participated in the post-trial follow-up for a median of 5.9 years (blood-pressure-lowering comparison) or 5.4 years (glucose-control comparison). Between-group differences in blood pressure and glycated hemoglobin levels during the trial were no longer evident by the first post-trial visit. The reductions in the risk of death from any cause and of death from cardiovascular causes that had been observed in the group receiving active blood-pressure-lowering treatment during the trial were attenuated but significant at the end of the post-trial follow-up; the hazard ratios were 0.91 ( $95 \%$ confidence interval [CI], 0.84 to 0.99 ; $\mathrm{P}=0.03$ ) and 0.88 (95\% CI, 0.77 to 0.99; $\mathrm{P}=0.04$ ), respectively. No differences were observed during follow-up in the risk of death from any cause or major macrovascular events between the intensive-glucosecontrol group and the standard-glucose-control group; the hazard ratios were 1.00 ( $95 \%$ CI, 0.92 to 1.08 ) and 1.00 (95\% CI, 0.92 to 1.08), respectively.

\section{CONCLUSIONS}

The benefits with respect to mortality that had been observed among patients originally assigned to blood-pressure-lowering therapy were attenuated but still evident at the end of follow-up. There was no evidence that intensive glucose control during the trial led to long-term benefits with respect to mortality or macrovascular events. (Funded by the National Health and Medical Research Council of Australia and others; ADVANCE-ON ClinicalTrials.gov number, NCT00949286.) 
P OST-TRIAL FOLLOW-UP STUDIES INVOLVing patients with diabetes have previously shown long-term beneficial effects of earlier periods of intensive glucose control, but not blood-pressure lowering, on a range of outcomes, including mortality and macrovascular events. ${ }^{1-3}$ The Epidemiology of Diabetes Interventions and Complications (EDIC) study, an extension of the Diabetes Control and Complications Trial (DCCT) involving young patients with type 1 diabetes and no history of cardiovascular disease, hypertension, or hypercholesterolemia, showed a lower risk of macrovascular events, as well as a sustained benefit with respect to microvascular complications, beyond the period of intensive glucose control. ${ }^{1}$ The post-intervention follow-up of the United Kingdom Prospective Diabetes Study (UKPDS) also showed long-term beneficial effects of intensive glucose control in patients with newly diagnosed type 2 diabetes. ${ }^{2}$ Among patients formerly assigned to intensive therapy as compared with conventional therapy, the reduced risk of microvascular events was maintained, and previously nonsignificant estimates of the effect of intensive therapy on the end points of death and myocardial infarction became significant with extended follow-up. ${ }^{2}$ In contrast, no long-term benefits were detected with improved blood-pressure control in the UKPDS. ${ }^{3}$

The Action in Diabetes and Vascular Disease: Preterax and Diamicron Modified Release Controlled Evaluation (ADVANCE) trial assessed the effects of routine blood-pressure lowering and intensive glucose control in a broad cross section of patients with type 2 diabetes. ${ }^{4,5}$ Routine administration of a single-pill (fixed-dose) combination of perindopril and indapamide was associated with a reduction in the risk of the primary composite end point of major macrovascular or microvascular events. Reductions in the risks of death from any cause, death from cardiovascular causes, and nephropathy were also observed. ${ }^{4}$ Intensive glucose control was associated with a reduction in the risk of the primary composite end point of major macrovascular or microvascular events, owing primarily to a reduction in the incidence of new or worsening nephropathy. ${ }^{5}$ This benefit with respect to nephropathy included a reduction in the incidence of end-stage renal disease but not of death from renal disease. ${ }^{6}$ No clear protective or harmful effects of intensive glucose control with respect to death from any cause or major macrovascular events were identified. ${ }^{5}$ We now report the results from the posttrial follow-up of the ADVANCE-Observational Study (ADVANCE-ON), which was designed to test the hypotheses that there would be long-term benefits of the two active interventions.

\section{METHODS}

\section{RANDOMIZED TRIAL}

Details of the recruitment of patients and the study design and methods have been published previously. ${ }^{7}$ In brief, 11,140 patients, 55 years of age or older, with type 2 diabetes and at least one additional risk factor for cardiovascular disease were enrolled between 2001 and 2003 at 215 centers in 20 countries. The study had a 2-by-2 factorial design. Patients were randomly assigned to a single-pill (fixed-dose) combination of perindopril (4 mg) and indapamide $(1.25 \mathrm{mg}$ ) or matching placebo, after a 6 -week active run-in period, and were also randomly assigned to a gliclazide (modified release)-based intensive glucose-control regimen, targeted to achieve a glycated hemoglobin level of $6.5 \%$ or lower, or to standard glucose control, with targets and regimens based on local guidelines. There were no inclusion or exclusion criteria related to blood pressure, and no blood-pressure targets were specified. The use of concomitant treatments during the trial, including other blood-pressure-lowering and glucosecontrol therapy, was at the discretion of the responsible physician. The last trial visits for the randomized blood-pressure-lowering comparison were completed in June 2007 after a median follow-up period of 4.4 years, at which time patients resumed their usual care for blood-pressure control. ${ }^{4}$ The randomized glucose-control regimen continued for an additional 6 months, to ensure adequate study power in the context of a smaller-than-anticipated separation in glycated hemoglobin levels between the groups. The last trial visits for the glucose-control comparison were completed in January 2008 after a median followup period of 5.0 years. ${ }^{5}$ At this time, all the patients discontinued their randomly assigned intervention and returned to the care of their usual physician for all aspects of treatment.

\section{POST-TRIAL FOLLOW-UP}

ADVANCE-ON was a post-trial follow-up study involving all surviving patients from the ADVANCE 


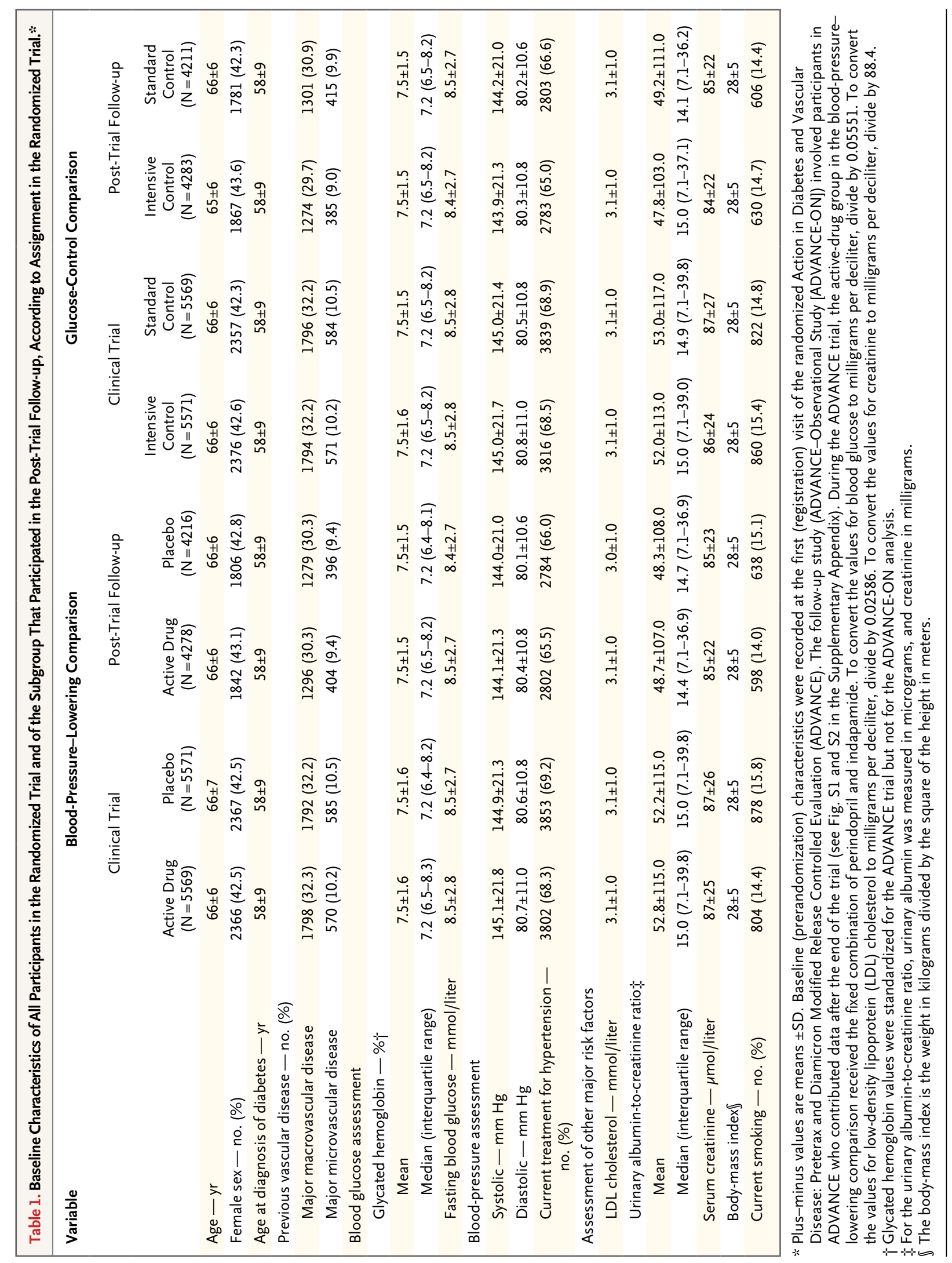


trial. A detailed description of the original study protocol has been published previously, and the current protocol, including the statistical analysis plan (which was completed before the end of the follow-up period), is available with the full text of this article at NEJM.org. ADVANCE-ON was an investigator-initiated study that was designed, conducted, analyzed, and interpreted independently of the funders, including the commercial sponsor (Servier International). Servier International was given the opportunity to comment on the final draft of the manuscript but had no role in the decision to submit the manuscript for publication. The first two authors wrote all drafts of the manuscript. The writing committee (i.e., all the authors) and the management committee (see the Supplementary Appendix, available at NEJM.org), neither of which included representatives of the sponsors, had final responsibility for the manuscript and for the decision to submit it for publication.

Two years after completion of the final ADVANCE trial visits, all local trial sites were invited to participate in the follow-up study, and 172 of $215(80 \%)$ agreed. After approval of the study by the ethics review board at each site, all surviving trial patients were invited to participate in the post-trial follow-up. In January 2010, annual post-trial visits commenced. At the first post-trial visit, all the participants provided written informed consent and completed a standardized questionnaire on the occurrence of all study outcomes of interest and all medications they were taking. A random subgroup of $2000 \mathrm{pa}-$ tients, balanced across regions and across the prior randomized study groups, were also invited to undergo assessment of the glycated hemoglobin level, fasting blood glucose level, blood pressure, weight, serum creatinine level, and urinary albumin-to-creatinine ratio at the first post-trial visit, to determine whether differences observed during the trial (in-trial period) persisted. For patients known to have died after the final in-trial visit, the cause and date of death were recorded. For patients unwilling or unable to attend study visits in person, follow-up assessments were conducted by telephone or home visit, or information was provided by the primary care physician, other health care providers, or next of kin. At annual visits, patients completed a questionnaire on medication taken and study outcome events. In addition, at the final visits, which occurred between January 1, 2013, and February 28, 2014, patients attending visits in person were invited to undergo an assessment of the glycated hemoglobin level, fasting blood glucose level, weight, blood pressure, serum creatinine level, and urinary albumin-to-creatinine ratio, regardless of whether they had undergone these assessments at the first visit.

\section{STUDY OUTCOMES}

The two prespecified primary outcomes for the present study were death from any cause and major macrovascular events (a composite, as in the randomized trial, of nonfatal myocardial infarction, nonfatal stroke, or death from any cardiovascular cause). The prespecified secondary outcomes were death from cardiovascular causes, fatal or nonfatal myocardial infarction, fatal or nonfatal stroke, major clinical microvascular events (a composite of end-stage renal disease, defined as requirement for renal-replacement therapy; death from renal disease; requirement for retinal photocoagulation; or diabetes-related blindness in either eye), the separate components of this composite outcome, and major hypoglycemia (as defined in the original trial protocol ${ }^{5}$ ). It was not possible to replicate the outcomes, "major microvascular events" and "new or worsening nephropathy," as defined in the original trial, because levels of serum creatinine and urinary albumin were measured in only a subgroup of participants during the post-trial follow-up. Outcomes occurring during the post-trial follow-up period were as reported by investigators at the study centers, according to prespecified definitions and criteria, and were not centrally adjudicated.

\section{STATISTICAL ANALYSIS}

All analyses were performed according to the initial study-group assignment. Treatment effects were examined with the use of cumulative-incidence survival curves and Cox proportional-hazards models. Data were censored at the time of the first relevant end point, the date of the patient's death, the date of the patient's last visit (for those still alive), or, for patients whose vital status was unknown at the end of the study (February 28,2014$)$, the date the patient was last known to be alive. Hazard ratios were estimated for the in-trial period and over the entire period of follow-up according to the intention-to-treat principle. We also performed a nonrandomized, 


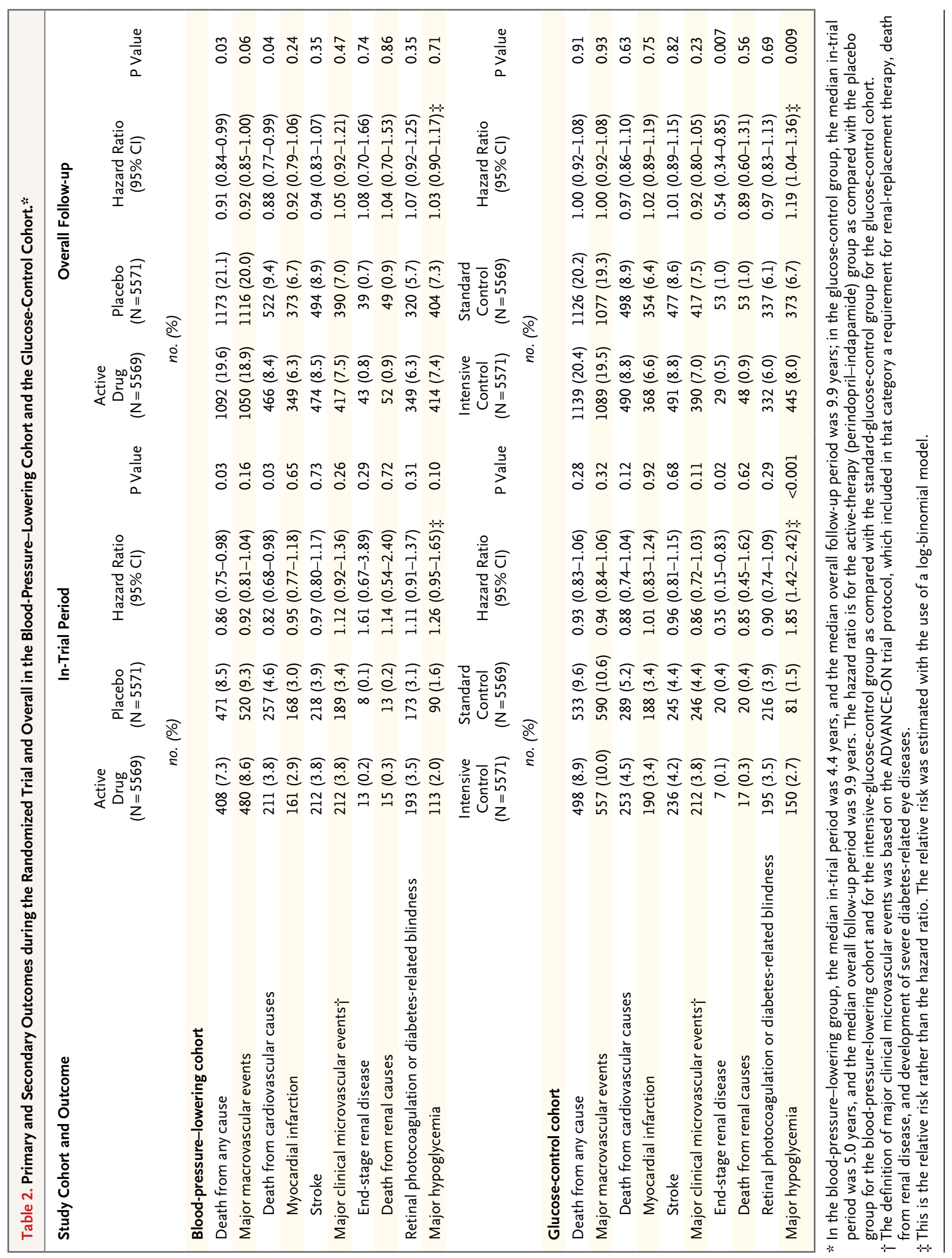


observational analysis of incident events during the post-trial period alone. Serial hazard ratios with $95 \%$ confidence intervals were estimated at the end of each calendar year of post-trial follow up. Each hazard ratio was obtained from a Cox model that included all the data collected up to the end of that calendar year. The interaction between the effects of intensive glucose control and blood-pressure lowering and the homogeneity of treatment effects in prespecified subgroups were tested by adding an interaction term to the relevant Cox models. A sensitivity analysis that included data only from sites that were able to follow at least $85 \%$ of surviving patients was performed for the entire period of follow-up.

The analyses were performed with the use of SAS software, version 9.2. All tests were two-sided, and $\mathrm{P}$ values of less than 0.05 were considered to indicate statistical significance. The protocol prespecified that no adjustments would be made for the multiple statistical testing. ${ }^{8}$ In light of this, the findings were interpreted with caution.

\section{RESULTS}

\section{FOLLOW-UP}

Of the 10,261 participants who were alive when the blood-pressure-lowering comparison was completed and the 10,082 patients who were alive when the glucose-control comparison was completed, 8494 (83\% and 84\%, respectively) enrolled in the post-trial follow up; 5131 of the 7279 patients who were alive at the end of the follow-up period $(70 \%)$ completed a visit during the final year of the follow-up study (Fig. S1 and S2 in the Supplementary Appendix). The first post-trial visits occurred a median of 3.5 years after the final trial visit for the blood-pressure-lowering comparison and 2.9 years after the final trial visit for the glucose-control comparison. The median in-trial, post-trial, and total follow-up periods were 4.4 years, 5.9 years, and 9.9 years, respectively, for the blood-pressure-lowering comparison and 5.0 years, 5.4 years, and 9.9 years, respectively, for the glucose-control comparison.

\section{CHARACTERISTICS OF THE PATIENTS}

The prerandomization characteristics of the entire trial cohort and of the cohort that contributed further data during the post-trial follow-up are shown according to the original study-group assignment; the characteristics were similar in the two cohorts apart from such changes as are consistent with a healthy-survivor effect in the post-trial cohort (Table 1). The prerandomization characteristics of the subgroups that had biochemical levels measured at the first and final post-trial visits were also similar to those of the entire cohort (Table S1 in the Supplementary Appendix). In addition, the prerandomization characteristics of the patients who completed a visit in the final year of post-trial follow-up were similar to those of patients who did not (Table S2 in the Supplementary Appendix).

\section{TREATMENT PATTERNS}

After completion of the blood-pressure-lowering comparison of the trial, the use of perindoprilindapamide, other blood-pressure-lowering therapies, and other medications was well balanced between the group that had originally been assigned to perindopril-indapamide and the group that had originally been assigned to placebo (Table S3 in the Supplementary Appendix). The use of blood-pressure-lowering therapies had decreased by the first post-trial visit and then increased by the final post-trial visit, although approximately $20 \%$ of the patients remained off any such therapy.

After completion of the glucose-control comparison of the trial, the use of oral glucose-lowering therapies and insulin in the group that had originally been assigned to intensive glucose control and the group that had originally been assigned to standard glucose control converged, although some differences remained between the two groups (Table S4 in the Supplementary Appendix). The use of insulin increased more in the standard-control group than in the intensivecontrol group, whereas the use of sulfonylureas, including modified-release gliclazide, decreased in both groups over time.

\section{BLOOD PRESSURE AND GLYCEMIC CONTROL}

The mean between-group difference in blood pressure observed during the randomized ADVANCE trial $(5.6 / 2.2 \mathrm{~mm} \mathrm{Hg}, \mathrm{P}<0.001)^{4}$ was no longer evident 6 months after the end of that part of the trial: the blood pressures recorded at the time of the final randomized visit for the patients in the glucose-control comparison (6 months after the last visit for the blood-pressure control comparison) were $137 / 74 \mathrm{~mm} \mathrm{Hg}$ in the perindopril-indapamide group and 136/74 $\mathrm{mm} \mathrm{Hg}$ in the placebo 


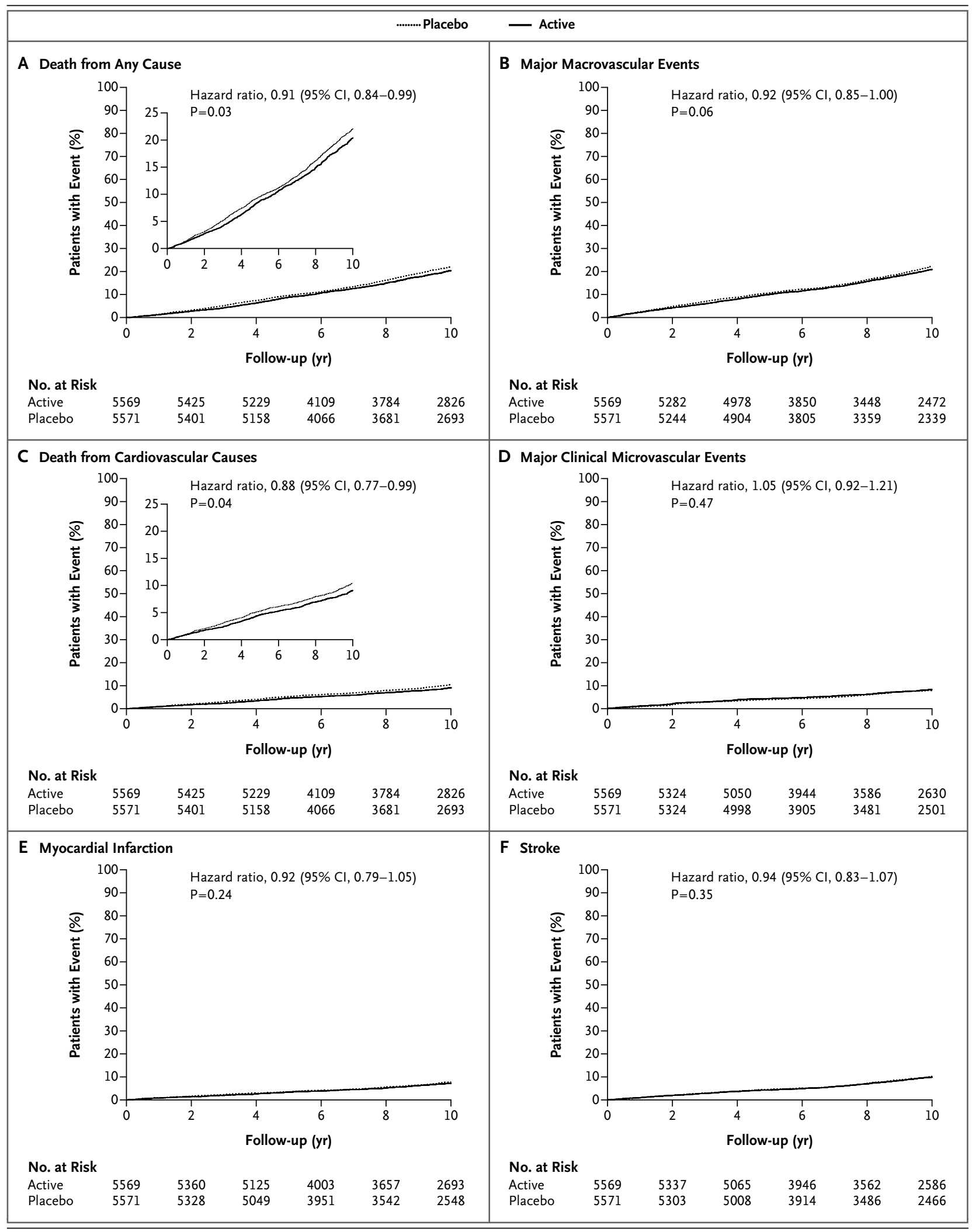

The New England Journal of Medicine

Downloaded from nejm.org at AUSTRALIAN NATL UNIV on May 7, 2015. For personal use only. No other uses without permission. Copyright @ 2014 Massachusetts Medical Society. All rights reserved. 
Figure 1 (facing page). Cumulative Incidence of Events, According to Blood-Pressure-Lowering Study Group.

Shown is the percentage of patients who had events at any time after the start of randomized treatment in the Action in Diabetes and Vascular Disease: Preterax and Diamicron Modified Release Controlled Evaluation (ADVANCE) trial, according to assignment to the active-drug (perindopril-indapamide) group or the placebo group. Cumulative hazard ratios (active-drug group vs. placebo group) and $\mathrm{P}$ values are shown for a 12 year period from the start of randomized treatment to the end of the post-trial follow-up in the ADVANCEObservational Study (ADVANCE-ON). The insets in Panels $A$ and $C$ (which show outcomes that were reduced significantly with the active drug) display the same data on an enlarged y axis.

group. The levels remained similar in the two blood-pressure-lowering study groups through the post-trial period (Table S5 in the Supplementary Appendix).

The mean between-group difference in glycated hemoglobin levels $(0.67$ percentage points, $\mathrm{P}<0.001)^{5}$ observed during the randomized ADVANCE trial was no longer evident by the first post-trial visit, an average of 2.9 years later ( 0.08 percentage points; $95 \%$ confidence interval $[\mathrm{CI}],-0.07$ to $0.22 ; \mathrm{P}=0.29$ ), and the levels remained similar at the conclusion of the posttrial follow-up $(7.2 \%$ in the intensive-therapy group and $7.4 \%$ in the standard-therapy group) (Table S6 in the Supplementary Appendix).

\section{OTHER RISK FACTORS}

Among the patients included in the blood-pressure-lowering comparison, the incidences of other risk factors were well balanced between the perindopril-indapamide group and the placebo group (Table S5 in the Supplementary Appendix). Among the patients included in the glucose-control comparison, the small difference of $1.6 \mathrm{~mm} \mathrm{Hg}$ in systolic blood pressure that had been observed, on average, between the two glucose-control groups during the trial was diminished and no longer significant at the first post-trial visit $(1.2 \mathrm{~mm} \mathrm{Hg}$, $\mathrm{P}=0.17)$ and the final post-trial visit $(0.9 \mathrm{~mm} \mathrm{Hg}$, $\mathrm{P}=0.14)$. The mean body weight, serum creatinine level, and urinary albumin-to-creatinine ratio were similar in the intensive and standard glucose-control groups at the final post-trial visit (Table S6 in the Supplementary Appendix).

\section{PRIMARY OUTCOMES}

During the randomized blood-pressure intervention, 879 patients died and 1000 patients had a major macrovascular event (Table 2). During the post-trial follow-up period, an additional 1386 patients died and 1166 patients had an incident major macrovascular event. Among patients assigned to perindopril-indapamide therapy, there was a significant but attenuated cumulative benefit with respect to the incidence of death from any cause that extended to the end of the overall follow-up period (hazard ratio, 0.91; 95\% CI, 0.84 to $0.99 ; \mathrm{P}=0.03$ ) (Table 2 and Fig. $1 \mathrm{~A}$ and $2 \mathrm{~A})-$ a finding consistent with the in-trial finding of a significant risk reduction of $14 \%$ in the rate of death from any cause among patients assigned to perindopril-indapamide therapy (hazard ratio, $0.86 ; 95 \% \mathrm{CI}, 0.75$ to $0.98 ; \mathrm{P}=0.03$ ). There was no evidence that the cumulative effects with respect to death from any cause varied according to the subgroups studied, including the subgroup defined according to assignment to intensive glucose control versus standard glucose control $(P>0.20$ for interaction for all subgroup analyses) (Fig. S3 in the Supplementary Appendix). There was no cumulative benefit of perindopril-indapamide with respect to major macrovascular events, and the hazard ratios for this composite outcome were similar at the end of the in-trial period and at the end of the overall follow-up period, although they were not significant at either time (Table 2 and Fig. $1 \mathrm{~B}$ and 2B).

During the randomized glucose-control intervention, 1031 patients died and 1147 patients recorded a major macrovascular event (Table 2). During the post-trial period, an additional 1234 patients died and 1019 patients recorded a major macrovascular event. There were no cumulative benefits of intensive glucose control with respect to either death from any cause or major macrovascular events (Table 2 and Fig. $3 \mathrm{~A}$ and $3 \mathrm{~B}$ and $4 \mathrm{~A}$ and $4 \mathrm{~B})$ - results that were consistent with in-trial findings. There was no evidence that the cumulative effects with respect to death from any cause varied according to the patient subgroups studied, including the subgroup defined according to assignment to active blood-pressurelowering therapy versus placebo $(\mathrm{P}>0.10$ for interaction for all subgroup analyses) (Fig. S4 in the Supplementary Appendix). 


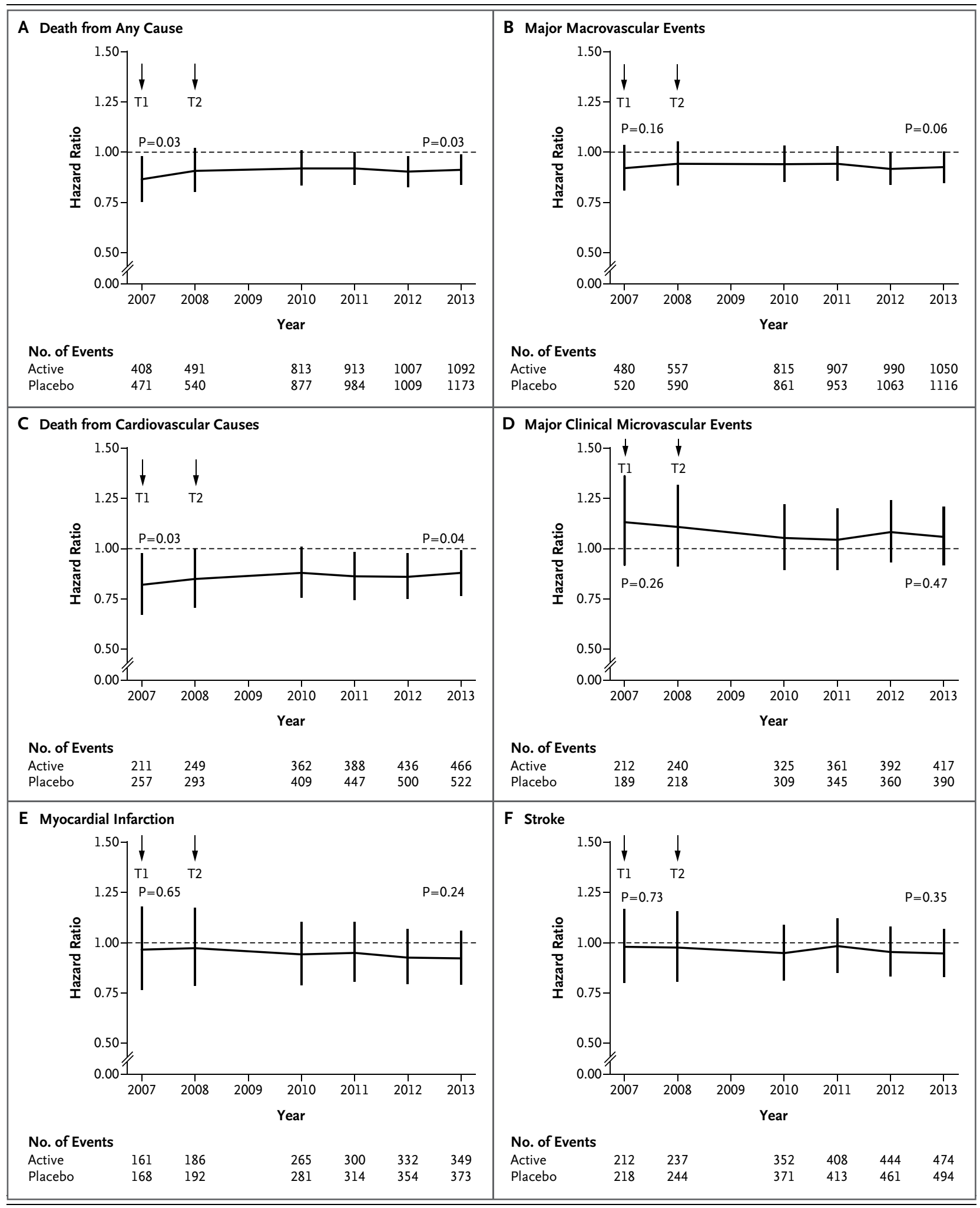

The New England Journal of Medicine

Downloaded from nejm.org at AUSTRALIAN NATL UNIV on May 7, 2015. For personal use only. No other uses without permission. 
Figure 2 (facing page). Hazard Ratios for Events, According to Blood-Pressure-Lowering Study Group.

Hazard ratios are shown for events that occurred from the start of randomized treatment to the end of the blood-pressuring-lowering comparison (2007), to the end of the glucose-control comparison (2008), and to the end of each year of post-trial follow-up (2010 through 2013). The hazard ratios are for the activedrug (perindopril-indapamide) group versus the placebo group. $P$ values are for the between-group comparison at the final visit for the randomized trial in 2007 and at the end of the post-trial follow-up period. The data for 2013 include those obtained in the first 2 months of 2014, when follow-up was terminated. $\mathrm{Tl}$ indicates the final visit for the blood-pressure-comparison cohort, and T2 the final visit for the glucosecomparison cohort. Vertical lines indicate $95 \%$ confidence intervals.

\section{SECONDARY OUTCOMES}

In the blood-pressure-lowering cohort, an additional 520 deaths from cardiovascular causes, 393 myocardial infarctions, and 538 strokes were recorded during the post-trial period (Table 2). The in-trial reduction in the risk of death from cardiovascular causes among those assigned to perindopril-indapamide (hazard ratio, 0.82; $95 \%$ CI, 0.68 to $0.98 ; \mathrm{P}=0.03$ ) was attenuated but remained significant at the end of the overall follow-up period (hazard ratio, $0.88 ; 95 \% \mathrm{CI}, 0.77$ to 0.99; $\mathrm{P}=0.04$ ) (Table 2 and Fig. $1 \mathrm{C}$ and $2 \mathrm{C}$ ). There were no cumulative benefits with respect to any other secondary outcome, including major clinical microvascular events (Table 2).

In the glucose-control cohort, an additional 349 major clinical microvascular events were recorded during the post-trial period (Table 2). There were no cumulative benefits with respect to major clinical microvascular events (Table 2 and Fig. 3D and 4D) or severe diabetes-related eye disease (Table 2 and Fig. 3F and 4F). There was a significant cumulative benefit with respect to end-stage renal disease (hazard ratio, 0.54; 95\% CI, 0.34 to $0.85 ; \mathrm{P}=0.007$ ) (Table 2 and Fig. $3 \mathrm{E}$ and $4 \mathrm{E}$ ), although relatively few events were recorded. There was no cumulative benefit with respect to death from renal disease or any other secondary outcome, including death from cardiovascular causes, myocardial infarction, and stroke (Table 2).
There was no significant interaction between the effects of glucose control and blood-pressure lowering with respect to any primary or secondary outcome $(\mathrm{P}>0.10$ for interaction for all comparisons). When the cumulative effects were examined with data only from sites that were able to follow at least $85 \%$ of their surviving patients, the findings were unchanged in the glucose-control cohort, and the pattern of the effects in the blood-pressure-lowering cohort remained similar (Table S7 in the Supplementary Appendix). However, the reduction in major macrovascular events observed in the perindoprilindapamide group, which was not significant in the total cohort $(\mathrm{P}=0.06)$ (Table 2$)$, did become significant when only sites that were able to follow at least $85 \%$ of their surviving patients were considered $(\mathrm{P}=0.03)$ (Table S7 in the Supplementary Appendix). Conversely, the reduction in death from cardiovascular causes, which was significant in the total cohort $(\mathrm{P}=0.04)$, became nonsignificant when only sites that were able to follow at least $85 \%$ of their surviving patients were considered $(\mathrm{P}=0.06)$.

When the post-trial observational period was examined alone, there was no reduction in the risk of any outcome among patients assigned to perindopril-indapamide as compared with those assigned to placebo or among patients assigned to intensive glucose control as compared with those assigned to standard glucose control (Table S8 in the Supplementary Appendix). Although the rate of major hypoglycemia was low overall, the increase in that rate in the intensive-glucosecontrol group versus the standard-glucose-control group, which was significant during the trial, was not significant at the end of the posttrial follow-up, when only the post-trial period was considered (Table S8 in the Supplementary Appendix).

\section{DISCUSSION}

After following the current cohort for a total of 10 years, including the in-trial period and the post-trial follow-up, we observed attenuated but still significant reductions in the rates of death from any cause and from cardiovascular causes resulting from the 4.5-year period of blood-pres- 


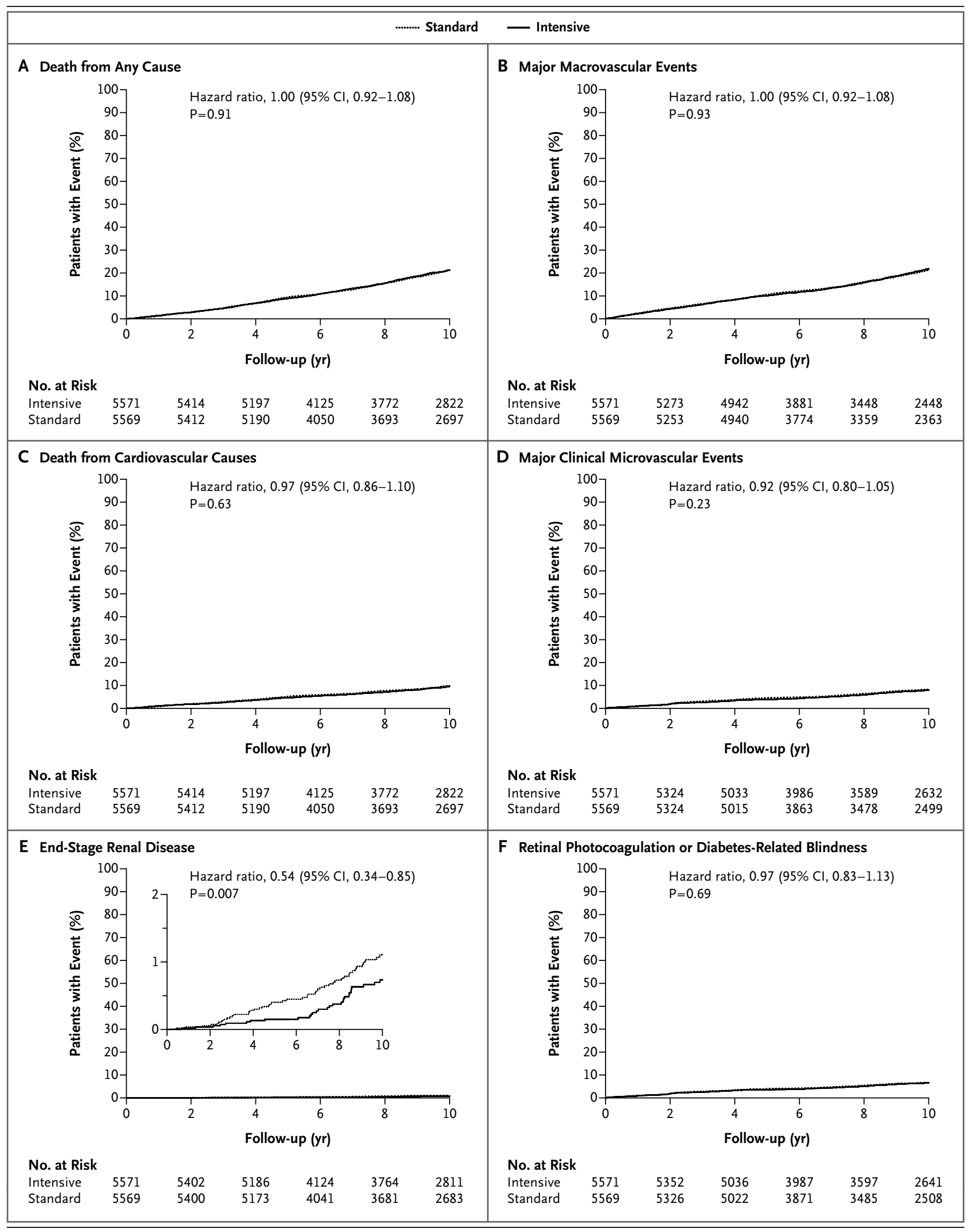

The New England Journal of Medicine

Downloaded from nejm.org at AUSTRALIAN NATL UNIV on May 7, 2015. For personal use only. No other uses without permission. Copyright (C) 2014 Massachusetts Medical Society. All rights reserved. 
Figure 3 (facing page). Cumulative Incidence of Events, According to Glucose-Control Study Group.

Shown is the percentage of patients who had events at any time after the start of randomized treatment, according to assignment to the intensive-glucose-control group or the standard-glucose-control group. Hazard ratios (intensive control vs. standard control) and $P$ values are shown for the 12 -year period from the start of randomized treatment to the end of the post-trial follow-up. The inset in Panel E (which shows an outcome that was reduced significantly with intensive glucose control) displays the same data on an enlarged y axis.

sure-lowering treatment with perindopril-indapamide (average difference in blood pressure of 5.6/2.2 $\mathrm{mm} \mathrm{Hg}$ between the perindopril-indapamide group and the placebo group during the original trial). In contrast, we did not observe any significant benefits with respect to mortality, macrovascular events, or microvascular events resulting from the 5-year period of intensive glucose control (average difference in glycated hemoglobin level of 0.67 percentage points between the intensive-glucose-control group and the standardglucose-control group during the original trial). When the prespecified components of the microvascular outcome were examined, we observed a persistent benefit of intensive glucose control with respect to end-stage renal disease but no new benefit with respect to serious eye complications.

The UKPDS post-trial follow-up study showed no persistence of the benefits of the earlier period of tight blood-pressure control with respect to macrovascular events or death. Although our blood-pressure findings appear to be different from those of the UKPDS, the point estimates for the major mortality end points are similar and are consistent with other post-trial follow-up studies of blood-pressure-lowering therapy in patients at high risk for cardiovascular events. ${ }^{9-13}$ Indeed, a comparison of in-trial and post-trial numbers of events suggests that the cumulative reductions in mortality in the perindopril-indapamide group can be ascribed largely to a carrying forward of the effects observed during randomized treatment. It is possible that with even longer post-trial follow-up these effects might have further dissipated, as occurred in the UKPDS. The carry-forward effect and the gradual attenuation of benefits over time reinforce the importance of continuing blood-pressure-lowering medications if the benefits of treatment are to be fully realized.

The DCCT-EDIC and UKPDS post-trial follow-up studies showed the long-term beneficial effects of earlier periods of intensive glucose control with respect to macrovascular events and death. ${ }^{1,2}$ We did not observe any such long-term benefits after post-trial follow-up. In our trial, the original benefits of intensive glucose control were due primarily to reductions in the incidence of new or worsening nephropathy, driven by reductions in the progression of albuminuria and serious renal disease requiring renal-replacement therapy. ${ }^{5,6}$ We were unable to obtain the biochemical measurements (serum creatinine level and urinary albumin-to-creatinine ratio) required to assess the outcome of new or worsening nephropathy in all patients who entered the posttrial follow-up, so any conclusions can be based only on certain components, such as end-stage renal disease or death from renal disease. We observed benefits with respect to end-stage renal disease but no effects on the rate of death from renal disease, which may reflect the persistence of the effects observed during the trial. It is possible that the small differences in blood pressure between the intensive-glucose-control group and the standard-glucose-control group during the trial and post-trial periods contributed up to one quarter of this beneficial effect, as was reported for the benefits observed in the original trial. ${ }^{5}$ Given the small number of events of end-stage renal disease (29 in the intensivecontrol group and 53 in the standard-control group), the benefits with respect to this end point should be interpreted with caution and studied further in future trials.

The divergent outcomes between our study and other studies of glucose control in patients with diabetes may be explained in part by differences in the response to the lowering of glucose across the trial populations. First, the younger patients with type 1 diabetes (in the DCCT-EDIC) ${ }^{1}$ or with newly diagnosed type 2 diabetes (in the UKPDS) ${ }^{2}$ may have been more likely to have long-term benefits from glucose lowering than the older patients with established disease who were included in our study. Second, there were differences between the studies in the in-trial levels of blood glucose, as reflected in the levels of glycated hemoglobin; the glycated hemoglobin level 


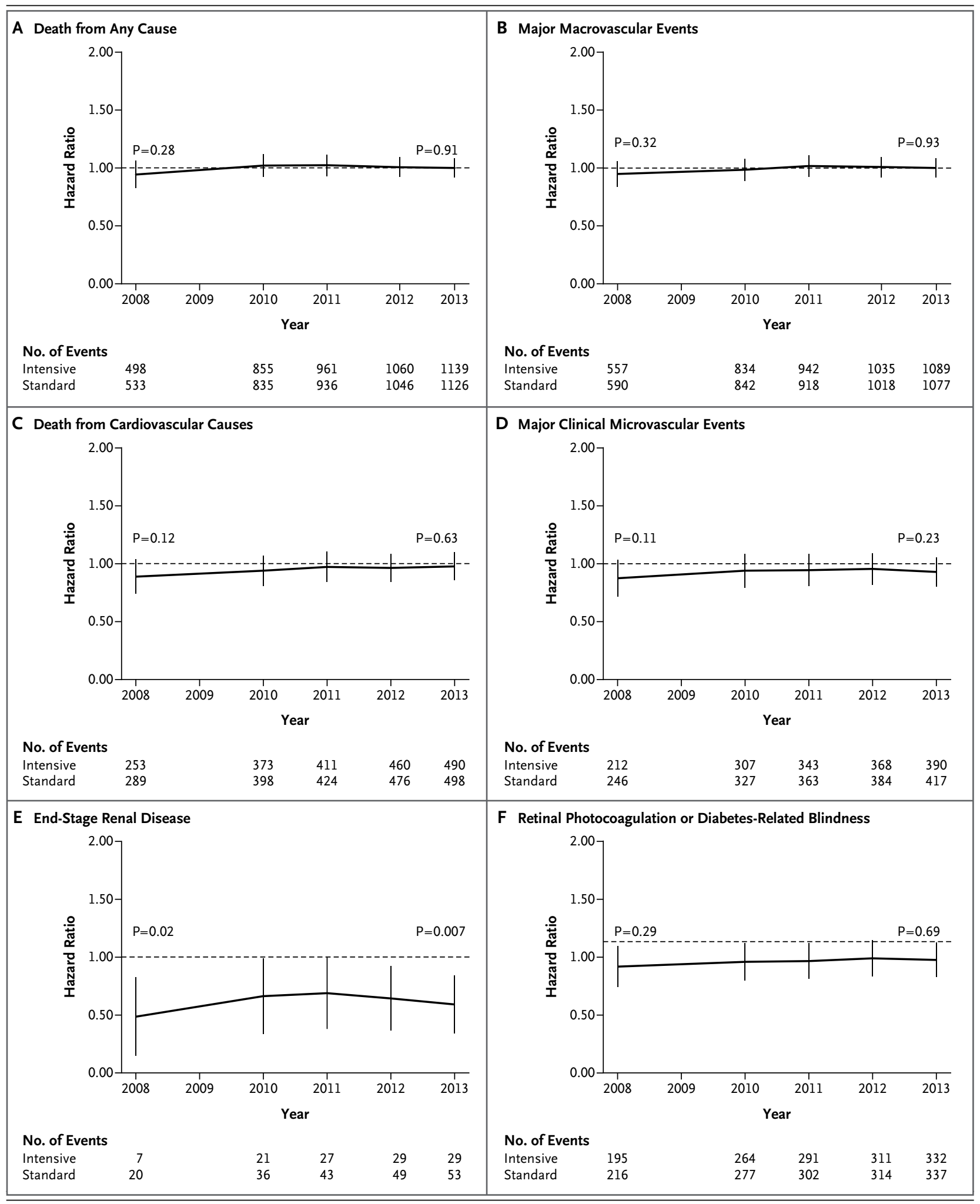

The New England Journal of Medicine

Downloaded from nejm.org at AUSTRALIAN NATL UNIV on May 7, 2015. For personal use only. No other uses without permission. 
Figure 4 (facing page). Hazard Ratios for Events, According to Glucose-Control Study Group.

Hazard ratios are shown for events that occurred from the start of randomized treatment to the end of the glucose-control comparison (2008) and to the end of each year of post-trial follow-up (2010 through 2013). The hazard ratios are for the intensive-control group versus the standard-control group; values less than 1.00 represent better outcomes in the intensive-control group. $P$ values are for the between-group comparison at the final visit for the randomized trial in 2008 and at the end of the post-trial follow-up period. The data for 2013 include those obtained in the first 2 months of 2014, when follow-up was terminated. Vertical lines indicate $95 \%$ confidence intervals.

differed between study groups by an average of 0.67 percentage points over a period of 5 years in the ADVANCE trial, but the between-group difference was much larger in the DCCT (2.0 percentage points over a mean of 6.5 years during the trial) and slightly larger in the UKPDS ( 0.9 percentage points over a median of 10 years during the trial). ${ }^{1,2,5}$ The baseline glycated hemoglobin levels in the patients in the DCCT and UKPDS $(>8.5 \%$ in both trials) were also much higher than the baseline level in the patients in the ADVANCE trial (7.5\%), ${ }^{1,2,5}$ Moreover, during posttrial follow-up in the UKPDS, ${ }^{2}$ the mean glycated hemoglobin level continued to decrease in both groups, whereas in our study, the level remained stable in the standard-glucose-control group and rose in the intensive-glucose-control group. Third, post-trial follow-up of our patients ( 5 years) was shorter than the follow-up for DCCT-EDIC and UKPDS ( $>10$ years for both trials) and may have been insufficient for benefits to emerge. Fourth, it is possible that more widespread use of effective background preventive therapy in the ADVANCE trial masked the long-term effects. Finally, competing risk, which is a greater issue among older patients than among younger patients, may not have allowed the full effects of the glucose intervention to be observed in our study.

Our post-trial analysis has some limitations. First, our findings must be considered in the context of incomplete follow-up of the total ADVANCE cohort. Nevertheless, patients from all the original study groups who did participate in the posttrial follow-up and those who completed a visit in the final year of follow up had baseline characteristics that were similar to those in the entire trial population, allowing for the healthysurvivor effect. Second, end points recorded during the post-trial follow-up were not adjudicated; however, we have previously shown that central adjudication in the trial had little effect on the observed hazard ratios for any outcomes. ${ }^{14}$ Third, many follow-up visits were conducted by telephone or questionnaire, with complete clinical and biochemical measurements available for only a limited subgroup of patients; therefore, we were not able to assess the possible persistence of benefits with respect to the original microvascular end point. Fourth, it should be stressed that although a comparison of outcomes across the entire follow-up period preserved the intention-to-treat principle, comparisons in the post-trial period alone are purely observational and hypothesisgenerating, because they may be confounded by differences in risk profiles arising during randomized treatment. Finally, given the multiple comparisons made, the results from individual hypothesis tests must be considered with caution.

In conclusion, among patients with longstanding type 2 diabetes, blood-pressure-lowering treatment with perindopril-indapamide for an average of 4.5 years resulted in attenuated but significant long-term benefits with respect to death from any cause and from cardiovascular causes, whereas intensive glucose control for an average of 5 years did not provide any long-term benefits with respect to death or major macrovascular events.

Supported by grants from the National Health and Medical Research Council of Australia (1006367, 358395, and 571281), a joint grant from Diabetes UK and the British Heart Foundation (28562), and an unrestricted educational grant from Servier International.

Dr. Zoungas reports receiving fees for serving on advisory boards from Merck Sharp and Dohme, Bristol-Myers SquibbAstraZeneca, Sanofi-Aventis, Novo Nordisk, and Amgen, lecture fees from Servier, Merck Sharp and Dohme, and BristolMyers Squibb-AstraZeneca, and fees to her institution for research contract work with Bristol-Myers Squibb-AstraZeneca; Dr. Chalmers, receiving lecture fees and travel support from Servier; Dr. Neal, receiving honoraria from Abbott, Novartis, Pfizer, Servier, and Roche and grant support from Roche, AbbVie, Janssen, and Dr. Reddy's Laboratories; Dr. Colagiuri, receiving fees for serving on advisory boards and lecture fees from Servier; Dr. Hamet, receiving consulting fees from Servier; Dr. Harrap, receiving lecture fees from Servier, Takeda, and Novartis; Dr. Heller, receiving fees for serving on advisory boards from Eli Lilly, Novo Nordisk, and Takeda and 
lecture fees from Eli Lilly, Novo Nordisk, Takeda, and Boehringer Ingelheim; Dr. Mancia, receiving lecture fees from Bayer, Boehringer Ingelheim, Daiichi-Sankyo, Medtronic, Novartis, Menarini International, Recordati, Servier, and Takeda; Dr. Marre, receiving personal fees from Novo Nordisk, Sanofi, Eli Lilly, Merck Sharp and Dohme, Abbott, Novartis, and AstraZeneca and grant support from Novo Nordisk, Sanofi, Eli Lilly, Merck Sharp and Dohme, and Novartis; Dr. Perkovic, receiving fees for serving on steering committees from AbbVie, Boehringer Ingelheim, Janssen, Vitae, and Astellas, fees for serving on advisory boards from Eli Lilly, lecture fees from AstraZeneca, Roche, and Merck, and grant support from Baxter, Janssen, and Novartis; Dr. Poulter, receiving honoraria from Servier, Takeda, Menarini, and Pfizer and grant support from Servier and Pfizer; and Dr. Williams, receiving lecture fees from Novartis, Boehringer Ingelheim, and Merck Sharp and Dohme. No other potential conflict of interest relevant to this article was reported.

Disclosure forms provided by the authors are available with the full text of this article at NEJM.org.

\section{APPENDIX}

The authors' full names and academic degrees are as follows: Sophia Zoungas, M.D., Ph.D., John Chalmers, M.D., Ph.D., Bruce Neal, M.D., Ph.D., Laurent Billot, M.Sc., Qiang Li, M.Biostat., Yoichiro Hirakawa, M.D., Ph.D., Hisatomi Arima, M.D., Ph.D., Helen Monaghan, B.Sc., Rohina Joshi, M.D., Ph.D., Stephen Colagiuri, M.D., Ph.D., Mark E. Cooper, M.D., Ph.D., Paul Glasziou, M.D., Ph.D., Diederick Grobbee, M.D., Ph.D., Pavel Hamet, M.D., Ph.D., Stephen Harrap, M.D., Ph.D., Simon Heller, M.D., Liu Lisheng, M.D., Giuseppe Mancia, M.D., Michel Marre, M.D., Ph.D., David R. Matthews, B.M., Ph.D., Carl E. Mogensen, M.D., Ph.D., Vlado Perkovic, M.D., Ph.D., Neil Poulter, M.D., F.Med.Sci., Anthony Rodgers, M.D., Ph.D., Bryan Williams, M.D., Ph.D., Stephen MacMahon, D.Sc., Ph.D., Anushka Patel, M.D., Ph.D., and Mark Woodward, Ph.D.

The authors' affiliations are as follows: the George Institute for Global Health (S.Z., J.C., B.N., L.B., Q.L., Y.H., H.A., H.M., R.J., V.P., A.R., S.M., A.P., M.W.) and Boden Institute (S.C.), University of Sydney, Sydney, the School of Public Health and Preventive Medicine, Monash University (S.Z.), Baker IDI Heart and Diabetes Institute (M.E.C.), and the University of Melbourne and Royal Melbourne Hospital (S. Harrap), Melbourne, VIC, and Bond University, Robina, QLD (P.G.) - all in Australia; the Julius Center for Health Sciences and Primary Care, University Medical Center Utrecht, and Julius Clinical Research, Utrecht, the Netherlands (D.G.); Research Centre, Centre Hospitalier de l'Université de Montréal, Montreal (P.H.); University of Sheffield and Sheffield Teaching Hospitals NHS Foundation Trust, Sheffield (S. Heller), Oxford Centre for Diabetes, Endocrinology and Metabolism (D.R.M.), and the George Institute for Global Health (S.M., M.W.), University of Oxford, Oxford, and the International Centre for Circulatory Health, Imperial College (N.P.), University College London (UCL) and the National Institute for Health Research UCL Hospitals Biomedical Research Centre (B.W.), London - all in the United Kingdom; the Chinese Hypertension League Institute, Beijing (L.L.); the University of Milan-Bicocca and Istituto Auxologico Italiano, Milan (G.M.); Hôpital Bichat-Claude Bernard and Université Paris 7, Paris (M.M.); Medical Department M, Aarhus Sygehus, Aarhus, Denmark (C.E.M.); and the Department of Epidemiology, John Hopkins University, Baltimore (M.W.).

\section{REFERENCES}

1. Nathan DM, Cleary PA, Backlund JY, et al. Intensive diabetes treatment and cardiovascular disease in patients with type 1 diabetes. N Engl J Med 2005;353:2643-53. 2. Holman RR, Paul SK, Bethel MA, Matthews DR, Neil HA. 10-Year follow-up of intensive glucose control in type 2 diabetes. N Engl J Med 2008;359:1577-89.

3. Holman RR, Paul SK, Bethel MA, Neil HA, Matthews DR. Long-term follow-up after tight control of blood pressure in type 2 diabetes. N Engl J Med 2008;359: 1565-76.

4. Patel A, MacMahon S, Chalmers J, et al. Effects of a fixed combination of perindopril and indapamide on macrovascular and microvascular outcomes in patients with type 2 diabetes mellitus (the ADVANCE trial): a randomised controlled trial. Lancet 2007;370:829-40.

5. Patel A, MacMahon S, Chalmers J, et al. Intensive blood glucose control and vascular outcomes in patients with type 2 diabetes. N Engl J Med 2008;358:2560-72.
6. Perkovic V, Heerspink HL, Chalmers J, et al. Intensive glucose control improves kidney outcomes in patients with type 2 diabetes. Kidney Int 2013;83:517-23.

7. ADVANCE Management Committee. Study rationale and design of ADVANCE: action in diabetes and vascular disease preterax and diamicron MR controlled evaluation. Diabetologia 2001;44:1118-20. 8. Schulz KF, Grimes DA. Multiplicity in randomised trials I: endpoints and treatments. Lancet 2005;365:1591-5.

9. Kostis WJ, Thijs L, Richart T, Kostis JB, Staessen JA. Persistence of mortality reduction after the end of randomized therapy in clinical trials of blood pressure-lowering medications. Hypertension 2010;56:1060-8.

10. Appel LJ, Wright JT Jr, Greene T, et al. Intensive blood-pressure control in hypertensive chronic kidney disease. N Engl Med 2010;363:918-29.

11. Beckett N, Peters R, Tuomilehto J, et al. Immediate and late benefits of treat- ing very elderly people with hypertension: results from active treatment extension to Hypertension in the Very Elderly randomised controlled trial. BMJ 2012;344: d7541.

12. Brouwers FP, Asselbergs FW, Hillege HL, et al. Long-term effects of fosinopril and pravastatin on cardiovascular events in subjects with microalbuminuria: ten years of follow-up of Prevention of Renal and Vascular End-stage Disease Intervention Trial (PREVEND IT). Am Heart J 2011;161:1171-8

13. Kostis WJ, Cabrera J, Messerli FH, et al. Competing cardiovascular and noncardiovascular risks and longevity in the Systolic Hypertension in the Elderly Program. Am J Cardiol 2014;113:676-81.

14. Hata J, Arima H, Zoungas S, et al. Effects of the endpoint adjudication process on the results of a randomised controlled trial: the ADVANCE trial. PLoS One 2013; 8(2):e55807.

Copyright (c) 2014 Massachusetts Medical Society. 